\title{
Análisis de los retornos a la educación de algunos oficios y operarios que laboran en el comercio al detal y de las grandes superficies ${ }^{1}$ Analysis of the Returns to Education of Some Trades and Operators that Work on Retail Trade and Large Areas
}

Recibido: 12 de mayo de 2015

Evaluado: 25 de junio de 2015 Aceptado: 6 de ahosto de 2015

\author{
Dustin Tahisin Gómez Rodríguez (Colombia) \\ Magíster en Estudio y Gestión del Desarrollo \\ Corporación Unificada de Educación Superior \\ dustin_gomez@cun.edu.co \\ dgomez@usbbog.edu.co \\ Héctor Mauricio Rincón Moreno (Colombia) \\ Magíster en Administración \\ Corporación Unificada de Educación Superior \\ mauricio_rincon@cun.edu.co
}

\section{Resumen}

El objetivo principal del siguiente artículo fue analizar los ingresos de los trabajadores del comercio al por menor y de grandes superficies en Colombia, desde la perspectiva del capital humano. La metodología es de corte cuantitativo, se utilizó ecuaciones mincerianas para estimar los retornos a la educación con los datos del DANE 2009-2013, y se aplicó el método de Heckman para reducir el sesgo de selección. La conclusión principal es que las ocupaciones y oficios que se pueden catalogar dentro del comercio al por menor y de grandes superficies tienden a

\section{Abstract}

The main objective of this article was to analyze the income of workers in the retail and supermarkets in Colombia, from the perspective of human capital. The methodology is quantitative court where Mincer equations are used to estimate the returns to education with data from DANE 2009-2013 as Heckman method was applied to reduce selection bias. The main conclusion is that the occupations and trades that can be categorized within the retail and supermarkets tend to get diminishing returns to education in the period.

1 Proyecto de investigación: La gerencia, la identidad y el merchandising del retail de bajo presupuesto, un nuevo modelo en pequeña y mediana superficies; realizado en conjunto con la Universidad de la ECCI, la Universidad Tecnológica de Bolívar (UTB), la Corporación Unificada de Educación Superior (CUN) y la Universidad Autónoma de México del Estado de Hidalgo. 
obtener retornos a la educación decrecientes en el periodo estudiado.

Palabras claves: capital humano, comercio al por menor, retornos a la educación.
Keywords: human capital, retail trade, returns to education. 


\section{Introducción}

La economía colombiana en el siglo XX ha tenido cambios vertiginosos: se pasó de estar en concordancia con el direccionamiento de la política de la Sustitución de importaciones, a una economía aperturista desde la década de 1990. En consecuencia, los consumidores, como receptores finales, tienen una mayor oferta de servicios y bienes que provienen de otras latitudes. En efecto, las empresas nacionales tanto del comercio al detal como de grandes superficies han tenido que buscar estrategias con las cuales puedan competir, sin desconocer que el sector de los hipermercados ha generado crecimiento considerable desde finales del siglo XX e inicios del siglo XXI (Silva, 2011). En el mismo sentido, se puede afirmar que el consumo del país crece paulatinamente a la vez que el comercio minorista nacional e internacional está ampliando sus expectativas y su portafolio. De ahí que se haya acrecentado las inversiones de grandes cadenas en Colombia, como Falabella, Almacenes Éxito y Carrefour, cuando este último estuvo en el país (G.R.N., 2010).

Sin embargo, a diferencia de estos crecimientos en la producción, los ingresos de los operarios no son de una gran cantidad de profesionales que trabajan en el aparato productivo colombiano evidencian brechas amplias en los retornos a la educación entre formales y no formales. De la misma forma ocurre entre hombres y mujeres de las mismas actividades económicas, oficios y ocupaciones, Isaza (2013), Isaza \& Reilly, (2011), Isaza (2003), (2002), Pabón, (2004), Barón (2010) y Farné (2006), sin desconocer las trasformaciones estructurales en el mercado laboral acontecidas desde el gobierno del expresidente Gaviria (1990-1994) y la ideología neoliberal (Gómez, 2013) que permea los planes de desarrollo.
En efecto, las discrepancias obedecen a las características propias del mercado laboral colombiano, en el que muchas de ellas no solo acontece en el país sino en muchos países de Latinoamérica, como es el caso de la segregación salarial por género (Galvis, 2010), (Bullon \& Rodes, 2004), (Garrido, 2010) y (Gaviria \& Székely, 2001).

Por otro lado, el capital humano es una teoría anclada en la escuela neoclásica de la economía con los aportes de los premios nobel Becker (2003), (1983), (1964), Becker et al. (1990), Mincer (1974), (1970), (1958) y Schultz (1961), (1960), quienes lo identifican como la acumulación de capacidades y experiencia que posee un individuo en el mercado laboral (Cardona et al., 2007). Allí distinguen dos maneras de formación: una denominada general, que se adquiere en el sistema educativo, y la específica, que se sostiene en el seno de la unidad de producción, sin desconocer la importancia de los hábitos de trabajo y la salud de las personas. En particular, el aprovechamiento de este capital humano se evidencia como una capital intangible, al que se le asigna un valor y es derrotero del crecimiento y el desarrollo económico de un país, así como de la competitividad de un sector de la economía (Valencia, 2005), (Behrman, 1999) y (Behrman et al., 1998). En efecto, la teoría del capital humano se sintoniza con entender la educación como una inversión que toma un individuo en un momento determinado, que es un sacrificio del consumo presente en aras de obtener en el futuro unos mejores ingresos, lo cual contribuye en el mejoramiento en conjunto de la sociedad, en virtud que al estar mayor cualificado se puede responder a los retos de un aparato productivo más complejo, dado la necesidad de adaptarse a las exigencias de un mundo que tiende a ser globalizado (Gómez, 2014b, 2013b). 
Por ello, la presente investigación realiza un breve análisis de los retornos a la educación de algunos oficios y operarios que laboran en el comercio al detal y de las grandes superficies que se pueden identificar en el CIIU (Clasificación Industrial Internacional Uniforme de todas las Actividades Económicas), y CIUO (Clasificación Internacional Uniforme de Ocupaciones). De igual manera, utiliza los datos de la GEIH (Gran Encuesta Integrada de Hogares) del Departamento Administrativo Nacional de Estadística, DANE, de Colombia, tomando los años comprendidos entre el 2009-2013, desde los postulados de la teoría del capital humano, en virtud de su aceptación en la economía laboral para poder identificar estos retornos.

\section{Metodología}

\section{Datos}

Los Microdatos son de la GEIH (Gran Encuesta Integrada de Hogares) del DANE, entre los años 2009 a 2013.

\section{Modelo Econométrico}

La ecuación de Mincer estima la reciprocidad que muestran las variables de Capital Humano sobre el logaritmo del ingreso laboral (Pabón, 2004).

$$
\begin{gathered}
\operatorname{Ln}(y)=\beta 0+\beta 1 s+\beta 2 \exp +\beta 3 \exp \mathrm{x}^{2}+\varepsilon \\
\operatorname{Ln}(y)=\beta 0+\beta 1 s+\beta 2 \exp +\beta 3 \exp \mathrm{x}^{2}+\varepsilon(1)
\end{gathered}
$$

En la que:

Y: ingresos del individuo.

S: número de años de educación formal.

Exp: número de años de experiencia laboral. $\varepsilon$ : término aleatorio que no puede explicar el modelo.

Para el modelo micro econométrico se realiza de la siguiente manera:

$$
\begin{gathered}
\text { Ln yi }=\beta 0+\beta 1 \text { expi }+\beta 2 \operatorname{expi}^{2}+\beta 3 \text { pri }+\beta 4 \mathrm{sec}+\beta 5 \text { uni }+\beta 6 \text { uni }+\beta 7 \text { ocu } 1 i \\
+\beta 8 \text { ocui }+\cdots b 8+k-1+\varepsilon i
\end{gathered}
$$

En la que:

Ln yi: ingreso de los asalariados en forma logarítmica.

Expi: experiencia del trabajador. $\operatorname{expi}^{\wedge}$ 2: experiencia del trabajador al cuadrado.

pri :nivel de primaria. sec: nivel de secundaria. uni: nivel universitario. ocu: ocupación y oficio. $\mathrm{k}$ : número de ocupaciones.

De igual manera, al modelo econométrico se le aplica la metodología propuesta 
por Heckman (1979), la cual permite la corrección de sesgo de selección si los individuos analizados no son una muestra aleatoria de la población objetivo (Gómez, 2014).

$$
\begin{gathered}
\ln (w f)=X f^{*} \beta f+\sigma f \lambda f+U f \ln (w f)=X f^{*} \beta f+\sigma f \lambda f+U f(3) \\
L n(W m)=X m^{*} \sigma m \lambda m+U m L n(W m)=X m^{*} \sigma m \lambda m+U m(4)
\end{gathered}
$$

En la que:

Wi: vector columna del logaritmo del salario por hora del individuo del sexo i.

$\mathrm{Xi}$ : matriz que contiene las características observadas de los individuos.

B: vector de coeficiente a estimar.

$\lambda$ : Término de corrección.

$\sigma$ : Covarianza entre los factores observables que afectan a la participación laboral de aquellos que influyen en el salario.

Ui: Término de perturbación aleatoria $\mathrm{E}$ $(\mathrm{u})=0^{2}$

\section{Resultados}

Las ocupaciones u oficios se tomaron desde CIIU (Clasificación Industrial Internacional Uniforme de todas las Actividades Económicas), y CIUO (Clasificación Internacional Uniforme de Ocupaciones). ${ }^{3}$ Con igual propósito, a las salidas se les realizó un promedio para obtener datos más robustos para el análisis (véase Isaza, 2013). En consecuencia, las estimaciones relacionadas con las variables de la teoría del capital humano reiteran lo que establece la literatura especializada, es decir, que a mayor número de años de experiencia y escolaridad se aumentan los ingresos de los asalariados. En igual sentido, los retornos a la experiencia potencial son significativos al $1 \%$, concordando con lo antes dicho, en virtud de que los ingresos laborales crecen y se desaceleran paulatinamente a largo plazo, es decir, en la vida laboral de la gran mayoría de las ocupaciones. En el mismo sentido, los coeficientes de género evidencian discriminación salarial en varias ocupaciones, ya que en igualdad de condiciones, en el mercado laboral colombiano los hombres poseen mayores retornos que las mujeres.

Por otro lado, los resultados demuestran que al realizar el método de Heckman (1979), tanto en la submuestra femenina como en la submuestra masculina, no hay sesgo de selección. En virtud de los coeficientes lambda, existe una correlación negativa estadísticamente significativa al $1 \%$, lo cual se puede interpretar como que las características no observadas por los datos -la habilidad y la motivación-, no tienen una carga representativa en los resultados (Gómez \& Rojas, 2014c).

2 Véase en (Gómez, 2014), (Gómez \& Rojas, 2014c) y (Gómez \& Barbosa 2013).

3 Ultima fecha de revisión: 8 de mayo de 2015 en https:// www.dane.gov.co/files/nomenclaturas/ciiu/CIIURev31AC.pdfy http://www.ilo.org/public/spanish/ bureau/stat/isco/ 
Tabla No. 1. Submuestra masculina

\begin{tabular}{|l|r|r|r|r|r|r|}
\hline \multicolumn{1}{|c|}{ Oficios y Ocupaciones } & $\mathbf{2 0 0 9} * *$ & $\mathbf{2 0 1 0} * *$ & $\mathbf{2 0 1 1}^{* * *}$ & $\mathbf{2 0 1 2}^{* * *}$ & $\mathbf{2 0 1 3}^{* * *}$ & promedio \\
\hline $\begin{array}{l}\text { Empleados de contabilidad, cajeros y } \\
\text { trabajadores asimilados }\end{array}$ & 0,2797 & 0,3133 & 0,3049 & 0,2797 & 0,2677 & 0,28906 \\
\hline $\begin{array}{l}\text { Supervisores de transporte y de } \\
\text { comunicaciones }\end{array}$ & 0,3215 & 0,2889 & 0,2816 & 0,3335 & 0,3215 & 0,3094 \\
\hline $\begin{array}{l}\text { Servicios de transportey de } \\
\text { comunicaciones }\end{array}$ & $-0,1284$ & 0,044 & $-0,1152$ & $-0,113$ & $-0,125$ & $-0,08752$ \\
\hline Gerentes (comercio al por mayory al detal) & 0,3065 & 0,3175 & 0,2874 & 0,3594 & 0,3474 & 0,32364 \\
\hline $\begin{array}{l}\text { Comerciantes propietarios (comercio al por } \\
\text { mayory al detal) }\end{array}$ & 0,1906 & 0,1852 & 0,1863 & 0,1384 & 0,1264 & 0,16538 \\
\hline $\begin{array}{l}\text { Supervisor de ventas } \\
\text { Vendedores, empleados de comercio y } \\
\text { trabajadores asimilados }\end{array}$ & 0,4234 & 0,4893 & 0,5905 & 0,5468 & 0,5588 & 0,52176 \\
\hline Servicios empresariales & 0,4586 & 0,4013 & 0,4894 & 0,4452 & 0,4332 & 0,44554 \\
\hline Agentes técnicos de ventas & N/D & 0,132 & 0,1277 & 0,1687 & 0,1807 & 0,152275 \\
\hline
\end{tabular}

Las estimaciones en promedio evidencian que para la submuestra masculina, los oficios y ocupaciones denominados supervisores de ventas y los comerciantes propietarios (comercio al por mayor y detal), obtienen los mayores retornos a la educación con 0,55 y 0,52 puntos logarítmicos respectivamente. De ahí que se pueda explicar estos altos retornos por ser cargos de mayor responsabilidad, los cuales, en promedio, necesitan de una mayor cualificación por parte del recurso humano que lo realiza. Uno de los puntajes más bajos es el serrvicio de transporte y de comunicaciones, con un promedio de - 0,08752 , lo que demuestra que en este oficio cada vez sus retornos son más decrecientes en virtud de que en ninguno de los años hubo un rendimiento positivo, lo cual concuerda con la dinámica de la industria colombiana que premia según la actividad económica la experiencia y la educación que tenga el individuo, sin olvidar el grado de competitividad del sector, ANIF (2012) y ANDI (2012).

Ahora bien, para las salidas en los retornos medios se encuentran los servicios empresariales con un 0,44 puntos logarítmicos y los gerentes al comercio por mayor $y$ al detal, 0,33, que se sintonizan con las caracterísiticas del aparato productivo colombiano y su mercado laboral, C.P.C. (2011-2012) y Salas-Velasco (2001). 
Tabla No. 2: Submuestra femenina

\begin{tabular}{|c|c|c|c|c|c|c|}
\hline Oficios y Ocupaciones & $2009 * * *$ & $2010 * * *$ & $2011 * * *$ & $2012 * * *$ & $2013^{* * *}$ & promedio \\
\hline $\begin{array}{l}\text { Empleados de contabilidad, cajeros y } \\
\text { trabajadores asimilados }\end{array}$ & 0,3037 & 0,3275 & 0,3148 & 0,3021 & 0,2901 & 0,30764 \\
\hline $\begin{array}{l}\text { Operadores de máquinas para cálculos } \\
\text { contables y estadísticos }\end{array}$ & 0,2498 & 0,1846 & 0,1959 & 0,2213 & 0,2333 & 0,21698 \\
\hline $\begin{array}{l}\text { Supervisores de transporte y de } \\
\text { comunicaciones }\end{array}$ & 0,438 & 0,3318 & 0,5681 & 0,6763 & 0,6883 & 0,5405 \\
\hline Servicios de transporte y de comunicaciones & 0,0334 & $-0,0899$ & $-0,2426$ & $-0,1197$ & $-0,1317$ & $-0,1101$ \\
\hline Gerentes (comercio al por mayor y al detal) & 0,2996 & 0,2519 & 0,245 & 0,3398 & 0,3748 & 0,30222 \\
\hline $\begin{array}{l}\text { Comerciantes propietarios (comercio al por } \\
\text { mayor y al detal) }\end{array}$ & $-0,1522$ & $-0,1644$ & $-0,1579$ & $-0,1902$ & $-0,1552$ & $-0,16398$ \\
\hline Supervisor de ventas & 0,6242 & 0,5157 & 0,5139 & 0,5205 & 0,5555 & 0,54596 \\
\hline $\begin{array}{l}\text { Vendedores, empleados de comercio y } \\
\text { trabajadores asimilados }\end{array}$ & 0,7095 & 0,6505 & 0,6156 & 0,574 & 0,539 & 0,61772 \\
\hline Servicios empresariales & 0,4279 & 0,4386 & 0,4757 & 0,4771 & 0,4891 & 0,46168 \\
\hline Agentes técnicos de ventas & 0,1821 & 0,2937 & 0,2696 & 0,2721 & 0,3071 & 0,26492 \\
\hline
\end{tabular}

Para la submuestra femenina, los datos indican que las supervisoras de transporte y de comunicaciones obtuvieron el mayor retorno con un promedio de 0,68 , seguidas de las vendedoras, empleadas de comercio y trabajadoras asimilados con $0,61, y$ las supervisoras de ventas con un promedio parecido de 0,54 puntos logarítmicos, en virtud de la coyuntura económica y de los imaginarios laborales de género frente a estos oficios y trabajos desde la perspectiva de la teoría de señalización, Barceinas et al. (2001) y Barón (2010). Desde el punto de vista de los retornos de educación medios, se enfatiza los servicios empresariales y gerentes con 0,4 y 0,3 puntos logarítmicos respectivamente. Para terminar, con los retornos de educación bajos, en los que los servicios de transporte y comerciantes propietarios reflejaron resultados negativos de $-0,11$ y -0,16, lo cual evidencia que son ocupaciones no alentadas entre las mujeres o con una tendencia negativa en retornos a la educación, Harmon (2001) y Hansen (2006).

\section{Discusión}

El estudio de Saboga \& Novoa (2011), concuerda con la presente inevstigación al concluir que los retornos a la educación tienden a ser más altos para la submuestra masculina que para la submuestra femenina, lo cual equivale a identificar la existencia de discriminación por género (Páez, 2012). En el mismo sentido,

... las regresiones de Mincer son formas de estimación muy conocidas en el campo de la economía laboral. Su utilidad es muy alta porque ha permitido -entre 
otras cosas- hacer medidas de las tasas de retorno privado y social de la inversión en capital humano, importantes para la toma de decisión en materia de política educativa. Estas estimaciones no han estado exentas de críticas por parte de los expertos, pero se siguen usando a falta de un mejor modelo de análisis. (Tenjo \& Herrera, 2009, p.16)

Lo anterior se puede dilucidar en los resultados presentados por la investigación, en la cual se muestra que a mayor cúmulo de años como de experiencia hay mayores aumentos en los ingresos, sin desconocer que el modelo se queda corto al hacer un comparativo específico de las ocupaciones y en catalogar un oficio como derrotero de trabajos que ofrece el comercio al detal y de las grandes superficies, López et al. (2011) y McConnell et al. (2003).

Por otro lado, las diferencias de estos trabajadores con otros asalariados son bastante amplias, si se comparara con investigaciones que tienen como diferenciador los grados de pregrado como de postgrado, Finnie \& Frenete (2003) y Forero \& Ramírez (2008), donde se identifica la necesidad de empoderar a estos individuos en su cualificación para mejorar sus ingresos y por consiguiente su calidad de vida, DANE (2011) y Davivienda (2012). Todavía más, los estudios sobre profesiones como los de Isaza (2013) y los de Gómez (2014), confirman que los profesionales poseen mayores retornos a la educación que las ocupaciones estudiadas, Arrow (1973), Medina (2009) y Mora (2003).

Por consiguiente, es imperativo resaltar la necesidad de cualificación del recurso humano como una inversión a corto plazo que puede representar mejores ingresos a mediano plazo, según las necesidades del aparato productivo colombiano y de su coyuntura. Sin olvidar que esto se puede materializar con la colaboración de las mismas empresas o por las motivaciones del individuo, dado que los datos del Observatorio Laboral de la Educación del Ministerio de Educación corroboran la relación lineal del concepto del capital humano frente a mayor número de años educativos y de experiencia, así como el aumento a mediano plazo de los salarios, sin desconocer que según la teoría y los datos, a largo plazo decrecerán estos mismos retornos desde el punto de vista de la vida laboral (O.L.E., 2012, 2011).

\section{Conclusiones}

Los cambios vertiginosos de la economía colombiana han posicionado el comercio al detal y el comercio en las grandes superficies como receptores de mano de obra calificada y no calificada. Lo que es más, aunque los salarios de varias ocupaciones y profesiones han mejorado paulatinamente, y otros oficios se han estacado según los resultados de las salidas del modelo, es evidente que existe segregación salarial en la gran mayoría de los oficios estudiados en el periodo y que es imperativo generar estrategias en las cuales estos individuos mejoren su calidad de vida, así como disminuir la brecha salarial entre géneros y entre ocupaciones para contribuir a una mayor equidad y justicia social.

Los retornos a la educación de los oficios y ocupaciones escogidos para la presente investigación evidencian diferencias contundentes tanto de género como entre la misma submuestra, lo cual refleja cómo los empleados que tienen un mayor grado de responsabilidad frente a las funciones propias de su cargo (gerentes, supervisores etc.), en promedio obtienen mejores retornos. Igualmente, se observa que en la 
submuestra femenina hay oficios y ocupaciones que tienen retornos negativos, lo cual tiene que ver con las características propias del oficio frente a los procesos sociales de la economía colombiana.

Una investigación que se puede bifurcar de la presente investigación sería una diversificación de los oficios en esta actividad económica. Sin embargo, lo que se pretendió realizar en la investigación fue identificar $y$ hacer un alto en el camino para poder interpretar los retornos a la educación de las ocupaciones más concatenadas con el comercio al detal y de las grandes superficies, en virtud del proyecto que pretende ser una amalgama de futuras investigaciones frente al tema.

\section{Referencias}

G.R.N. Global Retail Newsletter (2010). La letter de la Distribution Internationale, $X I(110)$.

ANIF. (2012). El comportamiento de la industria en 2011 y perspectiva. ANIF, 52-53. ANDI. (Diciembre de 2012). INFORME. Obtenido de Colombia: Balance 2012 y perspectiva 2013. Recuperado de: http:// www.larepublica.co/sites/default/files/larepublica/andi.pdf

Arrow, K. (1973). Higher Education as a Filter. Journal of Publics Economics.

2(3), 193-216.

Barceinas, F., Alonso, O., Bara, L. \& Roig, J. (2001). Hipótesis de señalización frente a capital humano. Revista Economía aplicada (en prensa). Recuperado de: http://www. etla.fi/PUE/REA.pdf.

Bara, J., Alonso, J. \& Roig, J. (2001). Hipótesis de señalización frente a capital humano. Revista de economía aplicada. (26), 125-147.

Barón, J. (2010). Primeras experiencias laborales de los profesionales colombianos: probabilidad de empleo formal y salarios. Documentos de trabajo sobre Economía regional. (132), 1-39.

Becker, G. (1983). El Capital Humano: un análisis teórico y empirico referido fundamentalmente a la educación. Madrid: Alianza editorial.

Becker, G. (1964). Human Capital. New York: Columbia University press.

Becker, G., Murphy, K. \& Tamura, R. (1990). Human Capital, fertility, and economic growth. NBER working papers No 3114. National bureau of economic research. Cambridge. 1-18

Becker, G. (2003). Capital Humano en la nueva sociedad. Presentación en la Fundación DMR. Recuperado de http://www.fundaciodmr.org/textos/conferencias01d.html

Behrman, J. (1999). Social mobility: Concepts and Measurement. En: New Markets, New opportunities?: Economic and Social Mobility in a Behrman, Jere R.

Behrman, J., Birdsall, N. \& Székely, M. (1998). Intergenerational schooling Mobility and Macro Conditions and Schooling Policies in Latin America. Working paper No 386. Inter-American Development Bank. Bullon, F. \& Rodes, C. (2004). Temporalidad y señalización en el mercado de trabajo: El papel de las empresas de trabajo temporal. Cuadernos de economía y dirección de la empresa. (18), 35-67.

Cardona, M., Montes, I., Vásquez, J., Villegas, M. \& Brito, J. (2007). Capital humano: Una mirada desde la educación y la experiencia laboral. Cuadernos de investigación, 1-40.

CIIU. (2015). Clasificación Industrial Internacional Uniforme de todas las Actividades Económicas. Recuperado de: https:// www.dane.gov.co/files/nomenclaturas/ciiu/ CIIURev31AC.pdf

CIUO. (2015) Clasificación Internacional Uniforme de Ocupaciones. Recuperado 
de http://www.ilo.org/public/spanish/bureau/stat/isco/

C.P.C. (2011-2012). Consejo privado de competitividad. Obtenido de Informe nacional de Competitividad: http://www.compite. com.co/site/wp-content/uploads/2011/11/ Resumen-ejecutivo.pdf.

DANE. (21 de Noviembre de 2011). Boletín de prensa. Recuperado de: http://www. dane.gov.co/files/investigaciones/boletines/ especiales/genero/bol_genero_2011.pdf

Davivienda. (2012). Informe de mercado laboral 2012. Recuperado de: https://www.davivienda.com/wps/wcm/ connect/e6842eb3-5c4a-474f-b2096e2d3229f8e7/Mercado+Laboral+2012.pdf ?MOD=AJPERES $\&$ CACHEID $=\mathrm{e} 6842 \mathrm{eb} 3-$ 5c4a-474f-b209-6e2d3229f8e7.

Farné, S. \& Vergara, C. (2008). Los Profesionales Colombianos en el siglo XXI, ¿̇más estudian, más ganan? Cuadernos de Trabajo (10).

Farné, E. (2006). El Mercado de Trabajo de los Profesionales Colombianos. Boletín del Observatorio del Mercado de Trabajo y la Seguridad Social, (9).

Finnie, R. \& Frenete, M. (2003). Earning differences by major field of study: evidence from three cohorts of recent Canadian graduates. Economics of Education Review, (22), 179-192

Forero, N. \& Ramírez, M. (2008). Determinantes de los ingresos laborales de los graduados universitarios durante el periodo 2001-2004. Serie documentos de trabajo, 1-53. Universidad del Rosario. Facultad de Economía.

Freire, M. \& Terjeiro, M. (2010). Las ecuaciones de Mincer y las tasas de rendimiento de la educación de Galicia. Capítulo 14, en: Investigaciones de Economía de la educación. (5), 285-304.

Galvis, L. (2010). Diferencias salariales por género y región en Colombia: Una aproximación con regresiones cuantiles. Documentos de trabajo sobre Economia regional. (131), 1-59.

Garrido, J. (2010). Rendimientos de la educación en Europa: un enfoque aplicado con variables instrumentales Recuperado de: https://www.usc.es/congresos/xiirem/ pdf/106.pdf

Gaviria, A. \& Székely, M. (2001). Intergenerational Mobility in Latin America. Documentos de trabajo (25). FEDESARROLLO.

Gómez, D. \& Barbosa, E. (2014). Situación laboral de los docentes colombianos desde la perspectiva del capital humano. 20092012. Tesis de grado de Maestría. Universidad de la Salle.

Gómez, D. (2014a). Análisis de los retornos a la educación de los ingenieros colombianos desde la perspectiva del capital humano (2009 - 2012). Revista Sinapsis (6), 3-15.

Gómez, D. (2014b). La competitividad. La paz y el ambiente regulatorio en Colombia. Revista opinión pública, (2), 34-41.

Gómez, D. \& Rojas, W. (2014c). Análisis de los ingresos de los técnicos y tecnólogos egresados de las Ciencias Administrativas y afines en Colombia desde la perspectiva del Capital Humano. 2001-2012. Revista TEKCNE, (13), 48-55.

Gómez, D. (2013). Posibles cambios estructurales para la implementación de los TLC en Colombia. Revista IGNIS, (6) 70- 83.

Gómez, D. (2013). La teoría del Capital Humano y sus críticas. Revista Dinámica empresarial, (2), 14-21.

Harmon, C., Walker, I., \& WestergaardNielsen, N. (2001) Education and earnings in Europe. A cross country analysis of return to education. Cheltenham: Edward Elgar

Hansen, J. (2006). Returns to university level education: Variations within disciplines, occupations and employment sectors. Canadá: Human Resources and Social Development. 
Heckman, J. (1979). Sample Selection Bias as a Specification Error. Econometrica, 47(1).153-161.

Isaza, J. (2013). Occupational segregation, gender wage differences and trade reforms empirical applications for urban Colombia. Tesis Doctoral, Departamento de Economía, Universidad de Sussex. Brighton, Reino Unido. Recuperado de: http://sro.sussex. ac.uk/44798/

Isaza, J., y Reilly, B. (2011) Selection effects, segregation and gender wage differences: evidence from Urban Colombia. Cuadernos de Economía de la Universidad de La Salle CE-03 11.

Isaza, J. (2003). Women workers in Bogota's informal sector: gendered impact of structural adjustmen policies in the 1990s. Tesis para optar el título de Magíster en Estudios de Desarrollo del Instituto de Estudios Sociales de The Hague-Holanda. Archivos de Economía.

Isaza, J. (2002). Women workers in Bogotás informal sector: Gendered impact of structural adjustment policies in the 1990s. Planeación y Desarrollo, (XXXIII), 411-468.

López, E., Ávila, A. \& Méndez, G. (2011). Dinámica del mercado laboral colombiano: un análisis de políticas de empleo aplicando dinámica de sistemas. Recuperado de: http://www.urosario.edu.co/urosario_files/ f2/f2b83f44-2e1e-4d71-878e-d8d53b106d94. pdf.

Mincer, J. (1974). Schooling, experience and earnings. New York: National Bureau of Economic Research.

Mincer, J, (1970). The distribution of labor incomes: A survey. Journal of economics literature. (8), 1-26.

Mincer, J, (1958). Investment Human capital and personal income distribution. Journal of political economy. (66), 281-302.
McConnell, C., Brue, S. \& MacPherson, D. (2003). Economía laboral. Madrid: Mc Graw Hill.

Medina, C. (2009). FEDESARROLLLO. Recuperado de: http://www.fedesarrollo.org. co/wp-content/uploads/2011/08/Debatesde-Coyuntura-Social-No-28.pdf.

Mortara, G. (1920). Lezioni di statistica económica e demográfica. Roma OECD (1998) Human capital investment. An international comparison. Paris: UNESCO Press.

Mora, J. (2003). Las ganancias de tener un título una aplicación al mercado laboral de Cali, 2000. Lecturas de Economía. 55-72 O.L.E. (20 de abril de 2012). Observatorio Laboral de la Educación. Capital Humano para el avance colombiano. Educación Superior Boletín 20. Recuperado de: http://www.mineducacion.gov.co/1621/ articles-92779_archivo_pdf_Boletin20.pdf. O.L.E. (2011). Observatorio Laboral de la Educación .Presentación de la Ministra de Educación, María Fernanda Campo Saavedra. Observatorio Laboral para la Educación: Seguimiento a los graduados de la educación superior en los últimos 10 años. Recuperado de: http://www.graduadoscolombia.edu.co/html/1732/articles 277950 presentacion_ministra.pdf.

Pabón, L. (2004). Universidad de los Andes. Facultad de Economía. Tesis de grado. Recuperado de: http://economia.uniandes. edu.co/estudiantes/premios/Premios_a_ los_mejores_trabajos/premios_a_los_mejores_trabhttp://economia.uniandes.edu. co/estudiantes/premios/Premios_a_los_ mejores_trabajos/premios_a_los_mejores_trabajos_2004.

Páez, I. (2012).Capital humano, redes externas e innovación en la industria colombiana. Revista estudios gerenciales. Edición especial. 81-107. 
Pons, E. (2001). Contraste de la hipótesis de señalización. Revista de educación. (326), 375-394.

Saboga, V. \& Novoa, S. (2011). Evolución de los diferenciales de ingresos laborales en los sectores formal e informal en el mercado de trabajo urbano en Colombia (1990-2004). Monografía. Bogotá: Universidad de la Salle.

Silva, H. (2011). Comportamiento de las superficies de retail en Colombia. Pensamiento y Gestión, 3-20.

Salas-Velasco, M. (2001). Determinantes salariales en el mercado laboral de los títulos universitarios. Murcia: X jornadas de la EDE. Universidad de Murcia.

Santamaría, M. (Julio de 2009). FEDESARROLLLO. Recuperado de: http:// www.fedesarrollo.org.co/wp-content/
uploads/2011/08/Debates-de-Coyuntura-Social-No-28.pdf

Schultz, T. W. (1961). Investment in human capital. The American economic review, 51(1), 1-17. Schultz. W. (1960). Capital formation by Education. The journal of political economy. 571-583.

Tenjo, J., \& Herrera, P. (Enero de 2009). Pontificia Universidad Javeriana. Facultad de Economía. Recuperado de: http://www. javeriana.edu.co/fcea/pdfs_depto_economia/paper_2009_02_dos_ensayos_sobre_ discriminacion.pdf.

Rodriguez, M. V. (2005). El capital humano, otro activo de su empresa. Entramado, 1(2), 20-33. 